\title{
Investigation of the impact of Photovoltaic Sizing and Siting Modeling on Micro-grids Energy Management Optimization
}

\author{
Marwa Abuelnasr \\ Electrical Power and Machines Department \\ Faculty of Engineering, Ain Shams University \\ Cairo, Egypt \\ marwa.mahmoud@eng.asu.edu.eg
}

\author{
Walid El-Khattam \\ Electrical Power and Machines Department \\ Faculty of Engineering, Ain Shams University \\ Cairo, Egypt \\ walid.elkhattam@eng.asu.edu.eg
}

\author{
Ibrahim Helal \\ Electrical Power and Machines Department \\ Faculty of Engineering, Ain Shams University \\ Cairo, Egypt \\ dr.i.helal@gmail.com
}

\begin{abstract}
The modeling of micro-grid's PV units' and loads' size and distribution along its network could severely affect the accuracy of power losses calculation and; thus the amount of energy to be imported from the main-grid to balance the load. Also, it will affect the micro-grid's optimal energy management results. Therefore, a comprehensive analysis is carried out to assess the impact of the PV's and the load's sizing and sitting (either lumped or distributed). Four case studies are carried out to illustrate the impact of the modeling on the micro-grid's losses and the imported energy from the main-grid and their costs. The obtained results are used to implement the proposed energy management two-single objective optimization functions applying Genetic Algorithm. Then, a fifth case study is carried out to optimize the micro-grid energy management process through an optimal chagrining - discharging scheduling of a storage module. The obtained results, recommendations, and evaluations for choosing a proper sizing and siting modeling and the chagrining discharging scheduling of a storage module under seasonal variations are reported and discussed.
\end{abstract}

Keywords- Energy management optimization, Genetic Algorithm optimization, Micro-grid.

\section{INTRODUCTION}

The integration of Distributed Energy Recourses (DER) such as wind turbines, Photovoltaic (PV), storage systems, and electric vehicles have increased the complexity of the distribution system's operation. To overcome this complexity, the electric energy consumption, the generation resources and energy storage operation should be optimally managed. This should be carried out in a way that insures distribution system's energy saving, improves energy efficiency, enhances system reliability, reduces greenhouse gas emissions, decreases fuel use and increase the use of Renewable Energy Sources (RES) while minimizing operating cost [1]. This

This work was supported in part by the short term fellowship of the Science \& Technology Development Fund (STDF), Ministry of Higher Education and Scientific Research, Egypt.. [ Project No.6463: Efficient Protection Systems for Microgrids.] energy management process is carried out through monitoring, controlling, and conserving energy in the whole distribution system.

Existing energy management systems can be divided into centralized and decentralized according to their philosophy of operation. The centralized energy management systems focus on controlling all energy activities in the whole system to reach an optimal energy management operation. However, their main disadvantage is reduced flexibility, as modifications are needed for each additional component (Generators, Storage systems...etc) to be installed in the system and the extensive computational requirements [2]. Due to this lack of flexibility, large numbers of DER in a system are difficult to be handled by the existing centralized control systems. It is difficult or nearly impossible to control the entire system by a single central controller. Thus, to avoid the centralized disadvantages research is being directed at micro-grids (decentralized energy control and management) because of their ability to enhance the reliability of a power system and reduce its environmental impact [3]. To divide existing bulky grids into micro-grids, electrical boundaries for sub-microgrids should hence be clearly defined: each micro-grid inside the defined boundaries should be controlled as a single entity that match its generation and load so that it can connect to or disconnect from the grid keeping the total load supplied [3].

Various research have been introduced on microgrid energy management based on decentralized energy management system. In [4], optimal allocation of DGs in the PV-based micro-grid was investigated after forecating the PV output power. The Modified Artificial Fish School algorithm was implemented on a cost function to optimaly manage energy in a PV based micro-grid. In [5], a nonlinear energy management system was applied to an islanded microgrid with storage system. Genetics Algorithm-based (GA) hybrid optimization algorithm was implemented on two levels in [6]. First, it 
applied nonlinear programming for modeling the energy management problem. Second, a hybrid solution was implemented to solve the microgrid energy management problem under study. A micro-grid with renewable and nonrenewable DGs was tested in [7] through a proposed distributed energy management strategy to reach the optimum energy management operation taking into consideration the distribution network constraints. In [8], the control of the power flow in the micro-grid was controlled in order to minimize power losses, energy cost, and dissatisfactions of the customers. It proposed a distributed energy management of micro-grids where both supply-side and demand side management were considered. In [9], a decentralized partiallyobservable Markov decision process was proposed to minimize operational costs of the micro-grid through coordinating the power production of DG sources and energy storage system. An energy management algorithm was presented in [10] in order to optimize the energy storage applications within micro-grids. While [11], GA was implemented to minimize energy cost and emissions of a micro-grid with different DGs and energy storage systems in both winter and summer seasons. However, it did not go into the details of each of the micro-grid's decision variables.

In this paper, the energy management problem of a PVenergy storage-based grid connected micro-grid is investigated through a proposed optimization process to minimize energy loss and cost. The analysis take into consideration; investigating the impact of sizing and sitting modeling of PV and load on the accuracy of the calculations, studying energy storage charging-discharging schedule, incorporating the impact of seasonal variations and customers behavioral changes on the demand.

\section{PROBLEM FORMULATION}

In this section, the problem under study's mathematical formulation; two objective functions and their associated operational constraints, is introduced. In addition, a detailed description of the system under study and the decision-making tool are presented.

\section{A. Mathematical Problem Formulation}

This section discusses the detailed mathematical formulation of energy management optimization problem under study.

\section{Objective Functions.}

\section{Objective Function 1:}

The first objective is to minimize the micro-grid energy loss that is calculated during the specified study period using its total number of hours, as follows [1]: $\mathrm{n}$ is the total number of buses,

$\mathrm{h}$ is the hour counter,

$h_{\text {tot }}$ is the total number of hours during the study period.

Objective Function 2:

The second objective is to minimize the total amount of energy received by the micro-grid from the main grid represented by its total energy cost. The total energy cost is given by the following:

$$
\text { Min. Cost }=a * \sum_{h=1}^{h_{\text {tot }}} c_{\text {grid }}^{h} x E_{\text {grid }}^{h}
$$

Where;

$\mathrm{a}$ is an adjustment factor

$\mathrm{c}_{\text {grid }}$ is the grid energy cost/hour

$\mathrm{E}_{\text {grid }}$ is the total amount of energy supplied by the grid

\section{Constraints}

The operation of the micro-grid under study is subject to the following constraints [1]:

- Power Balance Equations:

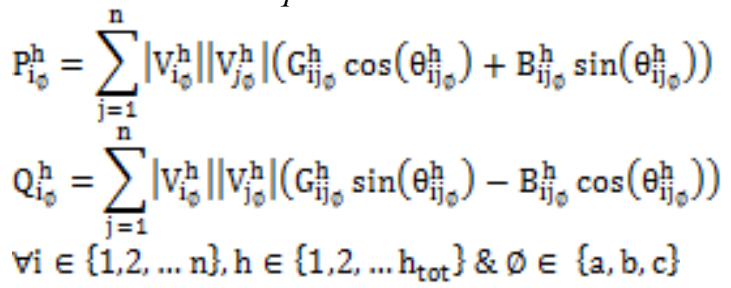

Where;

$P$ and $Q$ are the injected $\mathrm{kW}$ and $\mathrm{kVAr}$ respectively,

$B_{i j}$ is the susceptance in the bus admittance matrix that corresponds to the $\mathrm{i}^{\text {th. }}$ row and $\mathrm{j}^{\text {th. }}$ column,

$\theta_{i j}$ is the difference in voltage angles between the $\mathrm{i}^{\text {th }}$ and $\mathrm{j}^{\text {th. }}$ buses, and

$\emptyset$ is the phase number.

- Voltage Magnitude Limits [1]:

$V_{\min } \leq\left|\mathrm{V}_{\mathrm{i}_{\mathrm{a}}}^{\mathrm{h}}\right|,\left|\mathrm{V}_{\mathrm{i}_{\mathrm{b}}}^{\mathrm{h}}\right|,\left|\mathrm{V}_{\mathrm{i}_{\mathrm{c}}}^{\mathrm{h}}\right| \leq V_{\max }, \forall \mathrm{i} \in\{1,2, \ldots \mathrm{n}\}$

- Lines Thermal Limits [1]:

$\left|S_{j}^{h}\right|<\left|S_{j}^{h} \max ^{h}\right| \quad \forall i \in\left\{1,2, \ldots N_{L}\right\}$

Where;

$S_{j}$ is the line capacity

$N_{L}$ is the total number of micro-grid's lines.

- Storage model control tool:

The storage charging/discharging schedule is controlled by a controller that follows a load shape. The energy output of the storage model is expressed by:
Min. $\quad \mathrm{E}_{\text {loss }}=0.5 \mathrm{x} \sum_{\mathrm{h}=1}^{\mathrm{h}_{\text {tot }}} \sum_{\mathrm{i}=1}^{\mathrm{n}} \sum_{\mathrm{j}=1}^{\mathrm{n}}\left(\mathrm{G}_{\mathrm{ij}}^{\mathrm{h}} \mathrm{x}\left[\left(\mathrm{V}_{\mathrm{i}}^{\mathrm{h}}\right)^{2}+\left(\mathrm{V}_{\mathrm{j}}^{\mathrm{h}}\right)^{2}-2 \mathrm{xV}_{\mathrm{i}}^{\mathrm{h}} \mathrm{x}^{\top} E_{s}=E_{\text {stored }}+\sum_{h=1}^{h_{\text {tot }}^{-}} c_{s}^{\mathrm{h}} E_{s o}\right.\right.$

$V_{i}$ is the per unit voltage at bus $i$,

$G_{i j}$ is the conductance of the line connected between buses $i$ and $\mathrm{j}$,

$\delta_{\mathrm{i}} \& \delta_{\mathrm{j}}$ are the power angles of buses i \& j,
Where;

Estored is the initial stored $\mathrm{kWh}$ in the battery,

$E_{s o}$ is the storage rated $\mathrm{kWh}$,

$E_{s}$ is the total stored $\mathrm{kWh}$, and 
$c_{s i}^{h}$ is the load curve value for the storage at instant $h$.

\section{B. System Description}

The micro-grid test system is shown in Figure 1. It is part of the IEEE 123-bus three-phase unbalanced test system [12] according to an adequacy criteria explained in [3]. The microgrid is assumed to have scattered $1000 \mathrm{~kW}$ and $858 \mathrm{kVAr}$ load and five-solar based microFITs [13] rated at $700 \mathrm{~kW}$ and a storage unit rated at $250 \mathrm{~kW}, 1000 \mathrm{kWh}$. The tariff of the imported energy from the main-grid is calculated based on [14]. The micro-grid is assumed to be equipped with storage module with schedule control tool. The energy management system makes hourly generation plan for the next 24 hours to minimize the total operating cost based on the forecast power production of PVs and loads [2]. All PVs are assumed to work at unity power factor as the focus of this study is on the active power scheduling.

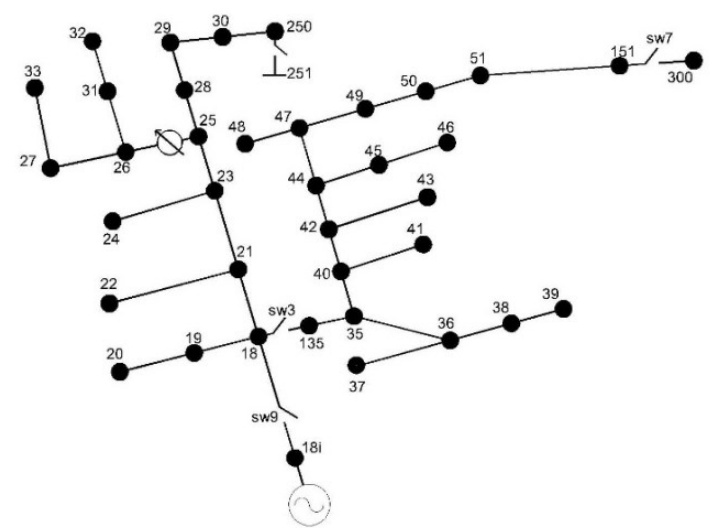

Figure 1 The micro-grid under study

\section{Decision-Making Tool}

The system under study is tested for single objective optimization technique each at a time. Genetic Algorithm (GA) was implemented to generate the required sets of solutions. GA relative to other Artificial Intelligence algorithm is less complex, more straightforward, more flexible, and offers a close-to-global optimum solution in a much shorter time. In the case of single objective, each chromosome is a proposed solution set (e.g. a charging or discharging of the storage model). If all constraints are satisfied, the system objective function is calculated and becomes the fitness value. These steps are repeated until a stopping criterion is reached. For this research, the stopping criterion is the generation number, which is provided by the operator.

\section{APPLICATIONS}

In this paper, four case studies are carried out to investigate the impact of modeling both load and PV (i.e. lumped and/or scattered) sitting and sizing on the value of the micro-grid's losses and energy received from the main grid as follows:

- Case study (1): A lumped load with a varying capacity lumped $\mathrm{PV}$ at the same bus

- Case study (2): A lumped load with a varying capacity lumped PV at two different buses
- Case study (3): Scattered loads with a varying capacity lumped PV

- Case study (4): The micro-grid with scattered loads and scattered $5 \mathrm{PVs}$

These analyses are carried out applying load flow analysis using OpenDSS software [15] representing a whole day (24 hours) of a typical day in the winter and a typical day in the summer. A stochastic data model, developed in [2], was utilized to forecast the load values and microFITs power output during the study periods.

After this investigation, for each season, the system is analyzed for two single-objective optimization problems namely minimizing the micro-grid's cost of; losses and energy received from the main grid. Case study (5) is carried out to optimization the micro-grid that poses scattered loads and scattered 5 PVs and an added 1 storage models. The storage module charging and discharging process is optimized to achieve the minimum loss and energy received from the main grid.

Case study (1): A lumped load with a varying capacity lumped $P V$ at the same bus

The system is simulated with a single lumped load (1000 $\mathrm{kW}$ and $858 \mathrm{kVAr}$ ) and a single lumped PV unit with varying capacity at the same bus, Bus 30 . For both summer and winter simulations results as in Tables 1.a and b respectively, the micro-grid's losses are decreased until a certain capacity of $\mathrm{PV}$ and then starts to increase again when the PV $\mathrm{kWh}$ is larger than that of the load. In the meanwhile, as the PV capacity increase, the amount of energy imported from the main grid decreases. Thus, the imported main grid energy's cost decreases. Also, it is shown that the PV capacity required in winter in more than that for summer due to the seasonal sun irradiance differences. Table I.b shows a negative cost that represents an export of energy from the micro-grid to the main-grid due to excessive PV generation than the micro-grid load. By comparing the losses between seasons, it is clear that the summer losses is higher than that of the winter as the summer loading is higher than the winter one as shown in Figures 2 and 3.

Table I.a Micro-grid losses and imported main grid energy cost for Case study (1) in winter

\begin{tabular}{|c|c|c|}
\hline \multirow{2}{*}{ PV (kW) } & \multicolumn{2}{|c|}{ Winter } \\
\cline { 2 - 3 } & Cost $(\$ / \mathrm{kWh})$ & Losses $(\mathrm{kWh})$ \\
\hline 0 & 1714.1 & 81 \\
\hline 500 & 1674.7 & 77 \\
\hline 1000 & 1582.7 & 73 \\
\hline 2000 & 1451.4 & 67 \\
\hline 3000 & 1320.4 & 64 \\
\hline 4000 & 1189.5 & 63 \\
\hline 5000 & 1058.8 & 64 \\
\hline 6000 & 928.4 & 68 \\
\hline 7000 & 798.1 & 73 \\
\hline
\end{tabular}


Table I.b Micro-grid losses and imported main grid energy cost for Case study

\begin{tabular}{|c|c|c|}
\hline \multirow{2}{*}{ PV (kW) } & \multicolumn{2}{|c|}{ Summer } \\
\cline { 2 - 3 } & Cost $(\$ / \mathrm{kWh})$ & Losses $(\mathrm{kWh})$ \\
\hline 0 & 2088.8 & 123 \\
\hline 100 & 2003.5 & 117 \\
\hline 200 & 1918.2 & 112 \\
\hline 300 & 1833.0 & 107 \\
\hline 400 & 1747.8 & 103 \\
\hline 500 & 1662.7 & 99 \\
\hline 1000 & 1237.7 & 85 \\
\hline 2000 & 391.7 & 88 \\
\hline 3000 & -449.7 & 129 \\
\hline
\end{tabular}

Figures 2 and 3 show the change of the PV capacity with respect to the load curve of the lumped load in both winter and summer seasons respectively.

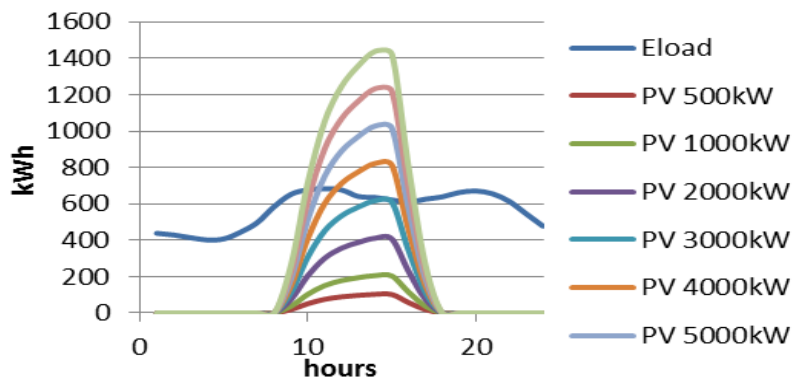

Figure 2 Case study (1): Micro-grid's PV and load energies in winter

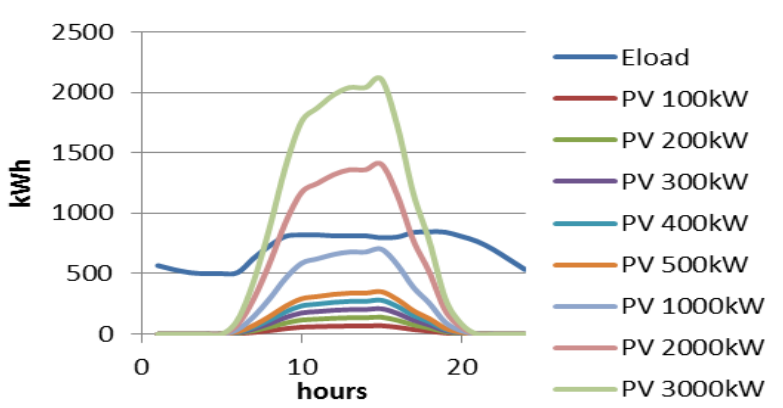

Figure 3 Case study (1): Micro-grid's PV and load energies in summer

Case study (2): A lumped load with a varying capacity lumped $P V$ at the different buses

Like in Case study (1), the simulation is carried out with PV installed at a different bus randomly, Bus 40, other than that of the load while keeping the same conditions in Case study (1) unchanged. It is realized that by increasing the PV capacity the losses kept constant until at a certain capacity of PV and then it start to increase. In the meanwhile, as PV capacity increases, the imported main-grid energy cost keeps dropping as shown in Tables II.a and b.

By comparing the results obtain in Tables 1 and 2, it is clear that having PV on different bus than the load will increase the load flow in the micro-grid and thus increases the losses and the amount of imported energy from the main-grid.
Table II.a Micro-grid losses and imported main grid energy cost for Case study (2) in winter

\begin{tabular}{|c|c|c|}
\hline \multirow{2}{*}{ PV(kW) } & \multicolumn{2}{|c|}{ Winter } \\
\cline { 2 - 3 } & Cost $(\$ / \mathrm{kWh})$ & Losses $(\mathrm{kWh})$ \\
\hline 0 & 1714.1 & 81 \\
\hline 400 & 1661.7 & 81 \\
\hline 500 & 1648.6 & 81 \\
\hline 1000 & 1583.1 & 81 \\
\hline 2000 & 1452.2 & 82 \\
\hline 3000 & 1321.3 & 85 \\
\hline
\end{tabular}

Table II.b Micro-grid losses and imported main grid energy cost for Case study (2) in summer

\begin{tabular}{|c|c|c|}
\hline \multirow{2}{*}{$($ PV kW) } & \multicolumn{2}{|c|}{ Summer } \\
\cline { 2 - 3 } & Cost $(\$ / \mathrm{kWh})$ & Losses $(\mathrm{kWh})$ \\
\hline 0 & 2088.8 & 123 \\
\hline 100 & 2003.8 & 123 \\
\hline 200 & 1918.9 & 123 \\
\hline 300 & 1834.0 & 123 \\
\hline 400 & 1749.0 & 124 \\
\hline 500 & 1664.1 & 125 \\
\hline
\end{tabular}

Figures 4 and 5 show the change of the PV capacity with respect to the load curve of the lumped load in both winter and summer seasons respectively.

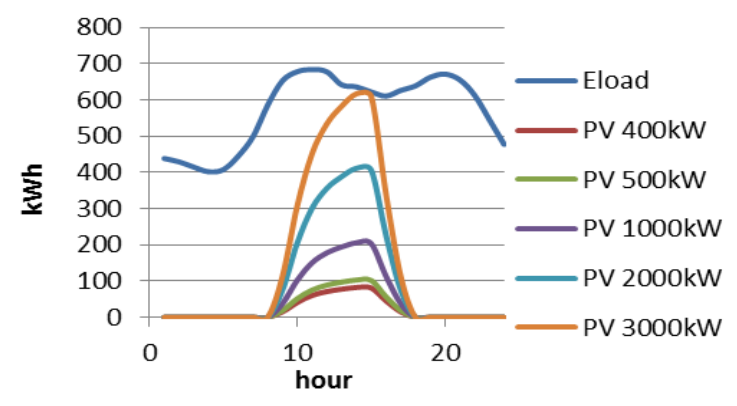

Figure 4 Case study (2): Micro-grid's PV and load energies in winter

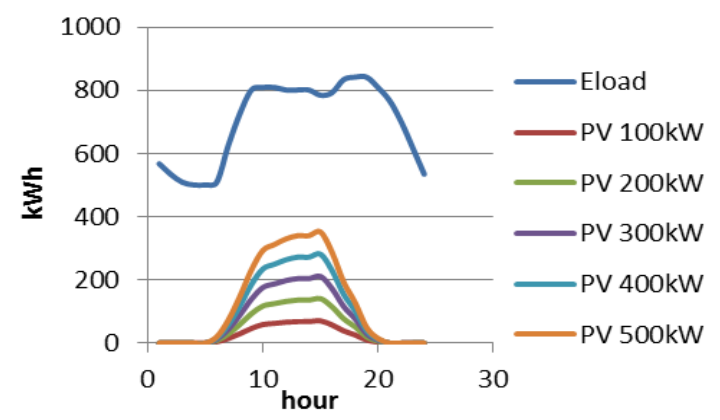

Figure 5 Case study (2): Micro-grid's PV and load energies in summer

Case study (3): Scattered loads with a varying capacity lumped PV

This simulation tests five scattered loads with same $\mathrm{kW}$ and kVAr as in Case study (1) installed on Buses-19, 28, 37,43 , and 50 while the PV is installed at Bus-40. Results shown in Tables III.a and $b$ indicate that the losses decrease then increase again, while the imported energy's cost is 
reduced as long as the PV capacity increases. Figures 6 and 7 show the change of the PV capacity with respect to the load curve of the total load in both winter and summer seasons respectively.

Table III.a Micro-grid losses and imported main grid energy cost for Case study (3) in winter

\begin{tabular}{|c|c|c|}
\hline \multirow{2}{*}{ PV kW } & \multicolumn{2}{|c|}{ Winter } \\
\cline { 2 - 3 } & Cost $(\$ / \mathrm{kWh})$ & Losses $(\mathrm{kWh})$ \\
\hline 100 & 1705.7 & 63 \\
\hline 500 & 1653.2 & 63 \\
\hline 1000 & 1587.6 & 62 \\
\hline 2000 & 1456.5 & 62 \\
\hline 3000 & 1325.5 & 62 \\
\hline 3500 & 1260.0 & 62 \\
\hline 4000 & 1194.5 & 63 \\
\hline
\end{tabular}

Table III.b Micro-grid losses and imported main grid energy cost for Case study (3) in summer

\begin{tabular}{|c|c|c|}
\hline \multirow{2}{*}{ PV kW } & \multicolumn{2}{|c|}{ Summer } \\
\cline { 2 - 3 } & Cost $(\$ / \mathrm{kWh})$ & Losses $(\mathrm{kWh})$ \\
\hline 100 & 2010.4 & 95 \\
\hline 500 & 1670.2 & 93 \\
\hline 1000 & 1245.3 & 93 \\
\hline 1100 & 1160.4 & 93 \\
\hline 1200 & 1075.5 & 94 \\
\hline 1300 & 990.6 & 94 \\
\hline 1400 & 905.8 & 95 \\
\hline 1500 & 820.9 & 96 \\
\hline 2000 & 397.0 & 104 \\
\hline
\end{tabular}

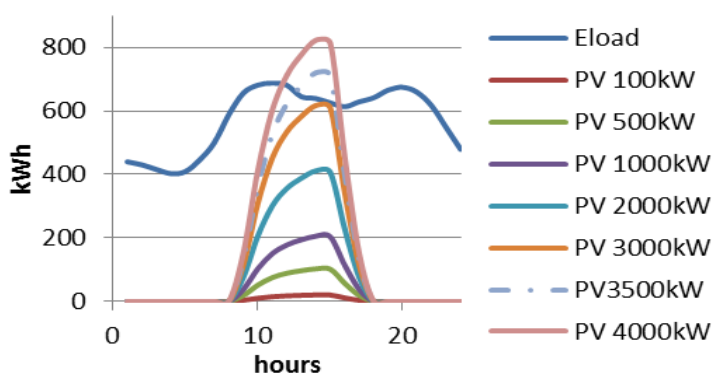

Figure 6 Case study (3): Micro-grid's PV and load energies in winter



Figure 7 Case study (3): Micro-grid's PV and load energies in summer
Case study (4 (Benchmark)): Scattered loads with a varying capacity scattered $P V$

The results obtain from this simulation showed almost similar results as in Case study (3).

From the above shown results obtain in Case studies (1) to (4), it can be concluded that near outputs can be achieved if the modeling of micro-grid loads and PV are scattered or lumped at the same bus. However, Case study (3) shows more accurate results than those of Case study (1). Therefore, the rest of the analysis in this paper will be carried on the concept of scattered loads and PV with a capacity that obtained in the winter season in Case study (3), i.e. $3500 \mathrm{~kW}$.

Case study (5): Optimization of the micro-grid with scattered 5 PVs and 1 storage module

The proposed two-single objective function optimization algorithm using GA technique is implemented to minimize the micro-grid losses and imported energy from the main-grid. This minimization is carried out through optimizing the storage module charging and discharging schedule. Tables IV.a and b show the optimal values in both seasons.

Table IV.a Optimized micro-grid losses and imported main grid energy cost for Case study (5) in winter

\begin{tabular}{|c|c|c|}
\hline \multirow{2}{*}{ PV kW } & \multicolumn{2}{|c|}{ Winter } \\
\cline { 2 - 3 } & Cost $(\$ / \mathrm{kWh})$ & Losses $(\mathrm{kWh})$ \\
\hline 0 & 1900.0 & 29 \\
\hline 3500 & 976.4 & 25 \\
\hline
\end{tabular}

Table IV.b Optimized micro-grid losses and imported main grid energy cost for Case study (5) in summer

\begin{tabular}{|c|c|c|}
\hline \multirow{2}{*}{ PV kW } & \multicolumn{2}{|c|}{ Winter } \\
\cline { 2 - 3 } & Cost $(\$ / \mathrm{kWh})$ & Losses $(\mathrm{kWh})$ \\
\hline 0 & 2319.5 & 44 \\
\hline 3500 & -1114.3 & 38 \\
\hline
\end{tabular}

In winter season, the optimization was able to reduce the losses and cost of imported energy by $60 \%$ and $23 \%$ respectively when compared with results obtained in Table IV.a. Similarly, in summer, the optimization was able to reduce the losses by $41 \%$ while and provides a saving by exporting energy to the main-grid by $200 \%$ when compared with results obtained in Table IV.b. Figures 8 and 9 show the obtained optimal results.

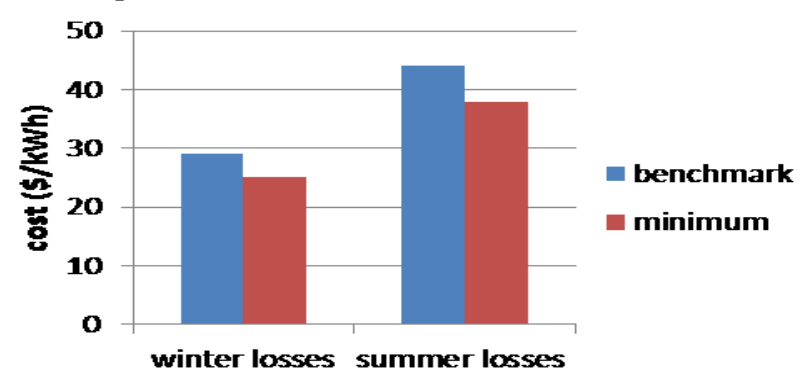

Figure 8 Optimization of the micro-grid's 




Figure 9 Optimization of the cost of the imported energy in winter and summer

\section{CONCLUSIONS}

The impact of sizing and sitting (scattered or distributed) modeling of a micro-grid's PV and loads was evaluated. Results show that under the current micro-grid's structure; the scattered modeling of PV and loads along the micro-grid's network provided near results of having a micro-grid's lumped PV and load installed at the same bus. However, modeling PV and load as lumped values at different busses reported far results. Therefore, accurate modeling will impact the calculated values of power losses as well as the amount of energy to be imported from the main-grid. This obtained results of PV and load sitting as well as the PV sizing that covers the micro-grid load under both winter and summer variations were used to optimally solve the micro-grid's energy management problem. A proposed two-single objective optimization functions via applying a GA is implemented to minimize the micro-grid losses and cost of imported energy from the main grid through optimal scheduling of charging and discharging of a storage module. Results show that the optimization was able to save a huge amount of micro-grid losses in both winter and summer. In the meanwhile, the optimization was able to reduce the amount of imported energy from the main-grid in winter, while was capable to export energy to the main-grid in summer through optimal charging - discharging scheduling of the storage module.

\section{REFRENCES}

[1] H. A. Mostafa, R. E. Shatshat and M. M. A. Salama, "Optimal Distribution Systems Operation Using Smart Matching Scheme (SMS) for Smart Grid Applications," IEEE Transactions on Smart Grid, vol. 5, no. 4, pp. 1938-1948, 2014.

[2] H. A. Mostafa, "Zonal Energy Management and Optimization System (ZEMOS) for Smart Grid Applications," Ontario, Canada, 2014.

[3] M. E. Nassar and M. M. A. Salama, "Adaptive SelfAdequate Microgrids Using Dynamic Boundaries," IEEE Transactions on Smart Grid, vol. 7, no. 1, pp. 105-113, 2016.

[4] Ke Huang; Xin Wang; Liming Wang, "Optimal energy management of gridconnected photovoltaic micro-grid," IEEE International Conference on Cyber Technology in
Automation, Control, and Intelligent Systems (CYBER), pp. 234-239, 2015.

[5] Zhiqiang Guo, Deshang Sha, and Xiaozhong Liao, "A Wireless, Decentralized and Nonlinear Energy Management Strategy Using PCC Frequency for Islanded AC Microgrids," IEEE Applied Power Electronics Conference and Exposition - APEC 2014, pp. $3136-3142,2014$.

[6] H. Li, C. Zang, P. Zeng, H. Yu and Z. Li, "A genetic algorithm-based hybrid optimization approach for microgrid energy management," Cyber Technology in Automation, Control, and Intelligent Systems (CYBER), 2015.

[7] W. Shi, X. Xie, C.-C. Chu and R. Gadh, "Distributed Optimal Energy Management in Microgrids," IEEE Transactions on Smart Grid, vol. 6, no. 3, pp. 1137 1146, 2015.

[8] W. Shi, X. Xie, C. C. Chu and R. Gadh, " Distributed Optimal Energy Management in Microgrids," IEEE Transactions on Smart Grid, vol. 6, no. 3, pp. 1137 1146, 2015.

[9] J. Wu and X. Guan, "Coordinated Multi-Microgrids Optimal Control Algorithm for Smart Distribution Management System," EEE Transactions on Smart Grid, vol. 4, no. 4, pp. 2174 - 2181, 2013.

[10]R. Zamora and A. K. Srivastava, "Energy management and control algorithms for integration of energy storage within microgrid," in Industrial Electronics (ISIE), 2014 IEEE 23rd International Symposium, 2014.

[11] M. Elsied, A. Oukaour, H. Gualous, R. Hassan and A. Amin, "An advanced energy management of microgrid system based on genetic algorithm," in Industrial Electronics (ISIE), 2014 IEEE 23rd International Symposium, 2014.

[12] "IEEE Distribution Planning Working Group Report, Radial Distribution Test Feeders," IEEE Transactions on Power Systems, vol. 6, 1991.

[13] [Online]. Available: http://microfit.powerauthority.on.ca/introduction

[14][Online]. Available: http://www.ieso.ca/powerdata/price-overview/hourly-ontario-energy-price

[15] [Online]. Available: https://sourceforge.net/projects/electricdss/ 